\title{
A pH-eQTL interaction at the RIT2-SYT4 Parkinson's disease risk locus in the substantia nigra
}

Authors and affiliations:

Sejal Patel ${ }^{1 *}$, Derek Howard ${ }^{1}$, Leon French ${ }^{1,2,3,4^{*}}$

1. Krembil Centre for Neuroinformatics, Centre for Addiction and Mental Health, Toronto, ON, Canada

2. Campbell Family Mental Health Research Institute, Centre for Addiction and Mental Health, Toronto, Canada

3. Department of Psychiatry, University of Toronto, Toronto

4. Institute for Medical Science, University of Toronto, Toronto, Canada

\section{Abstract}

Parkinson's disease (PD) causes severe motor and cognitive disabilities that result from the progressive loss of dopamine neurons in the substantia nigra. The rs12456492 variant in the RIT2 gene has been repeatedly associated with increased risk for Parkinson's disease. From a transcriptomic perspective, a meta-analysis found that RIT2 gene expression is correlated with $\mathrm{pH}$ in the human brain. To assess these $\mathrm{pH}$ associations in relation to $\mathrm{PD}$ risk, we examined the two datasets that assayed rs12456492, gene expression, and $\mathrm{pH}$ in the postmortem human brain. Using the BrainEAC dataset, we replicate the positive correlation between RIT2 gene expression and $\mathrm{pH}$ in the human brain $(n=100)$. Furthermore, we found that the relationship between expression and $\mathrm{pH}$ is influenced by rs12456492. When tested across ten brain regions, this interaction is specifically found in the substantia nigra. A similar association was found for the co-localized SYT4 gene. In addition, SYT4 associations are stronger in a combined model with both genes, and the SYT4 interaction appears to be specific to males. In the GTEx dataset, the $\mathrm{pH}$ associations involving rs12456492 and expression of either SYT4 and RIT2 was not seen. This null finding may be due to the short postmortem intervals (PMI) of the GTEx tissue samples. In the BrainEAC data, we tested the effect of PMI and only observed the interactions in the longer PMI samples. These previously unknown associations suggest novel mechanistic roles for rs12456492, RIT2, and SYT4 in the regulation of $\mathrm{pH}$ in the substantia nigra.

Keywords: RIT2, SYT4, pH, rs12456492, substantia nigra, Parkinson's disease 


\section{Introduction}

Parkinson's disease (PD) is a common neurodegenerative disease characterized by the loss of dopamine neurons in the substantia nigra. Individuals with PD show severe motor and cognitive disabilities. The etiology of PD is complex, with multiple genetic and environmental risk factors (Nalls et al. 2019; Bellou et al. 2016).

Recent GWAS studies of sporadic PD found that common genetic variants explain about $16-36 \%$ of PD's heritable risk (Nalls et al. 2019). Most studies have focused on genes that have been associated with both monogenic and sporadic forms of PD. These include SNCA, LRRK2, and GBA (Chang et al. 2017). However, over 90 independent risk signals have been identified. These recent GWAS hits are underexplored in the context of PD. For example, rs12456492 was first associated with PD in a 2011 GWAS study (Do et al. 2011). Subsequent studies have replicated this locus on chromosome 18, confirming an association with PD (Nalls et al. 2019). This locus contains the Ras Like Without CAAX 2 (RIT2) and Synaptotagmin 4 (SYT4) genes (Pankratz et al. 2012). The SYT4 gene is a member of the synaptotagmin family and regulates synaptic transmission (Dean et al. 2009). In the context of PD, Mendez et al. demonstrated that somatodendritic dopamine release depends on SYT4 (Mendez et al. 2011). However, the RIT2 and SYT4 genes have not been extensively characterized in relation to PD.

In a cross laboratory comparison of expression profiling data from normal human postmortem brains, Mistry and Pavlidis identified a robust correlation between RIT2 and tissue pH (Mistry and Pavlidis 2010). This meta-analysis included 11 studies that provided 421 cortical transcriptomes. In this meta-analysis, RIT2 was ranked tenth on the $\mathrm{pH}$ up-regulation list of 15,845 genes. The regulation of $\mathrm{pH}$ within the brain is crucial for proper physiological functioning. Specifically, impairment in this regulation can alter neuronal state leading to physiopathological conditions (Sinning and Hübner 2013). Results from the Mistry et al. meta-analysis suggest that RIT2 may be involved in neural $\mathrm{pH}$ regulation. Dysregulated $\mathrm{pH}$ can lead to oxidative stress and influence alpha-synuclein aggregation, which may play a role in PD pathology (Hwang 2013). These findings motivate a deeper characterization of $\mathrm{pH}$ and RIT2 gene expression in the context of PD.

In this study, we use the BrainEac data to replicate the correlation between RIT2 expression and $\mathrm{pH}$. In addition, we further test for associations between SYT4 and $\mathrm{pH}$. We characterize interactions involving a co-localized genetic risk variant for PD, $\mathrm{pH}, \mathrm{RIT2}$ and SYT4 gene expression. We explore these interactions in two independent postmortem brain datasets and test the impact of PMI and sex. We also perform co-expression searches to associate genes of known function to RIT2 and SYT4.

\section{Methods}

\section{BrainEac dataset}

Phenotype, genome-wide expression, and genotype information were obtained from The Brain expression quantitative trait loci (eQTL) Almanac (BrainEAC) project. This data from the UK Brain 
expression consortium was generated to investigate genetic regulation and alternative splicing. The consortium assayed genome-wide expression in ten brain regions using Affymetrix Exon 1.0 ST arrays (Illumina, San Diego, CA, USA) from 134 neuropathologically normal donors (Ramasamy et al. 2014). We extracted genotype data and expression values for the RIT2, SYT4 and CA10 genes from the BrainEAC web-based resource (http://www.braineac.org/). Age, sex, postmortem interval (PMI), RNA integrity number (RIN) and pH data were obtained from Gene Expression Omnibus (GSE46706) (Edgar, Domrachev, and Lash 2002). Of the 134 brains, we restricted our analyses to the 100 brains from the Medical Research Council (MRC) Sudden Death Brain and Tissue Bank in Edinburgh, UK (Millar et al. 2007) that had pH and genotype data. For each brain, $\mathrm{pH}$ was measured in the lateral ventricle because it is known to be stable across brain regions (Trabzuni et al. 2012). All ten regions were not sampled in all the brains, resulting in 73 substantia nigra samples. The average age of the cohort was 51.86 , with $76.71 \%$ composed of males.

\section{GTEx dataset}

The Genotype-Tissue Expression (GTEx) project was designed to identify eQTL's in diverse human tissues (Lonsdale et al. 2013). We extracted version 8 of GTEx data, which included expression data for the substantia nigra and genotype data for PD risk SNP rs12456492, resulting in a sample size of 113. Tissue samples were collected from non-diseased postmortem brain samples. RNA sequencing expression data was obtained from the GTEx Portal and log normalized (filename: GTEx_Analysis_2017-06-05_v8_RNASeQCv1.1.9_gene_tpm.gct). Genotype information was obtained from whole-exome sequencing by the GTEx consortium (filename:

GTEx_Analysis_2017-06-05_v8_WholeExomeSeq_979Indiv_VEP_annot.vcf). Additional information extracted from the GTEx Portal included age, sex, PMI, RIN and pH (measured in the cerebellum) (filename: GTEx_Analysis_v8_Annotations_SampleAttributesDS.txt). The average age of the cohort was 57.87 years, with $70.8 \%$ composed of males.

\section{Co-expression meta-analysis tools}

SEEK (Search-Based Exploration of Expression Compendium for Humans) was used to identify top genes co-expressed with RIT2. The top-ranked dataset from the co-expression result was downloaded to test to detail correlations between RIT2, SYT4 and CA10. Normalized expression data for GSE20146 was obtained from the Gemma web-based tool, using the filter option, which resulted in the removal of one outlier (GSM505262) (Lim et al. 2021).

\section{Statistical analysis}

Pearson correlation and ordinary least square linear models were performed in R (Core 2013).

\section{Availability of Code and Data}

Scripts and data for reproducing the majority of the analyses are publicly available online at https://github.com/Sejal24/PD_Manuscript_RIT2_SYT4_pH. The GTEx data is available via DBGap (Accession: phs000424.v8.p2). 


\section{Results}

\section{Brain-wide RIT2 and SYT4 gene expression is correlated with $\mathrm{pH}$}

To replicate the association between $\mathrm{pH}$ and $R I T 2$ and additionally test SYT4, we examined their relationships. The gene expression of RIT2 and SYT4 was averaged across all ten brain regions assayed in the BrainEAC study ( $\mathrm{n}=100$ brains). We observed a broad range of $\mathrm{pH}$ values (5.42 to 6.63). Gene expression and brain $\mathrm{pH}$ was correlated for RIT2 $(r=0.59, \mathrm{p}<0.0001)$, and SYT4 $(r=$ $0.58, \mathrm{p}<0.0001)$. As seen in Figure 1, five brains had abnormally low $\mathrm{pH}$ values, with $\mathrm{pH}$ values were lower than 2 standard deviations from the mean $(\mathrm{ph}<5.8)$. To prevent these outliers from skewing our downstream results, we have removed them from all subsequent analyses. After removal of these $\mathrm{pH}$ outlier brains, RIT2 remains correlated with $\mathrm{pH}(\mathrm{r}=0.22, \mathrm{p}<0.04)$, however SYT4 was no longer correlated with brain $\mathrm{pH}(S Y T 4: \mathrm{r}=0.14, \mathrm{p}=0.17)$. In agreement with Mistry et al., we also observe a correlation between postmortem brain $\mathrm{pH}$ and RIT2 gene expression.

Although $\mathrm{pH}$ was measured at the lateral ventricle, it is known that $\mathrm{pH}$ is relatively consistent brain-wide (Trabzuni et al. 2011). However, we tested if the correlation with gene expression varies across the ten brain regions profiled. For each individual brain region, RIT2 was most correlated with $\mathrm{pH}$ in the thalamus $\left(\mathrm{n}=90, \mathrm{r}=0.29, \mathrm{p}=0.005, \mathrm{p}_{\mathrm{FDR}}=0.055\right)$ and substantia nigra $(\mathrm{n}=70, \mathrm{r}$ $\left.=0.27, p=0.026, p_{\mathrm{FDR}}=0.13\right)$. In contrast, the correlation between SYT4 expression and $\mathrm{pH}$ was not statistically significant in any of the 10 regions. SYT4 correlation was high but not significant in the thalamus ( $r=0.20, p=0.057$ ). White matter and regions enriched for white matter (putamen and medulla) had the lowest $\mathrm{pH}$ correlations for RIT2 gene expression, whereas the medulla and substantia nigra had the lowest $\mathrm{pH}$ correlation for SYT4.
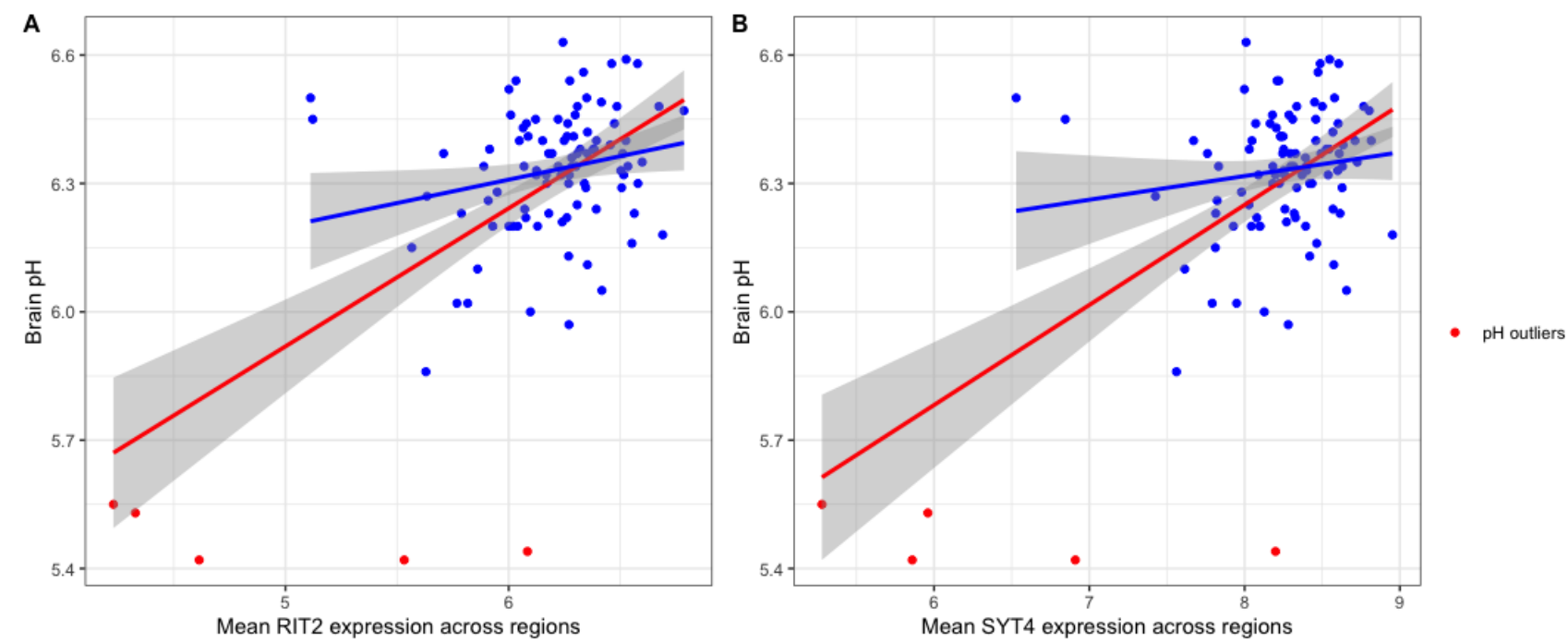

Figure 1. Scatter plots of the relationship between $\mathrm{pH}$ with RIT2 and SYT4 gene expression. $\mathrm{pH}$ outliers are colored red. Lines representing linear fits with (blue) and without outliers (red) include shaded areas marking the 0.95 confidence intervals. 


\section{Rs12456492 influences the association between RIT2 and SYT4 expression and $\mathrm{pH}$ in the substantia nigra}

Given that the single nucleotide polymorphism (SNP) rs12456492 lies between RIT2 and SYT4 is associated with Parkinson's disease, we tested if it is associated with brain $\mathrm{pH}$. Genotype at rs12456492 by itself was associated with brain $\mathrm{pH}(\mathrm{B}=0.06, \mathrm{p}=0.043)$. We next tested if this neighbouring PD risk variant influenced the correlations between gene expression and $\mathrm{pH}$. As depicted in Figures 2 and 3, an interaction between $\mathrm{pH}$, rs12456492 and either RIT2 or SYT4, was observed in the substantia nigra (RIT2: $\mathrm{B}=-0.15, \mathrm{p}<0.007, \mathrm{p}_{\mathrm{FDR}}<0.07$, SYT4: $\mathrm{B}=-0.16, \mathrm{p}<$ $\left.0.0001, \mathrm{p}_{\mathrm{FDR}}<0.001\right)$ but none of the other profiled regions. As shown in Table 1, after accounting for the covariates of sex, age, PMI and RIN, these signals remain. Compared to all other terms in the model, the $\mathrm{pH}$-eQTL interaction for either RIT2 or SYT4 expression is the most significant $(p<0.005)$ (Table 1). Specifically, in the BrainEAC data, individuals carrying the risk allele (AG and $\mathrm{GG}$ ), had a positive correlation between gene expression and $\mathrm{pH}$ but not in the AA genotype group. This suggests a weaker coupling between $\mathrm{pH}, \mathrm{RIT2}$ and SYT4 expression in the substantia nigra may be protective.
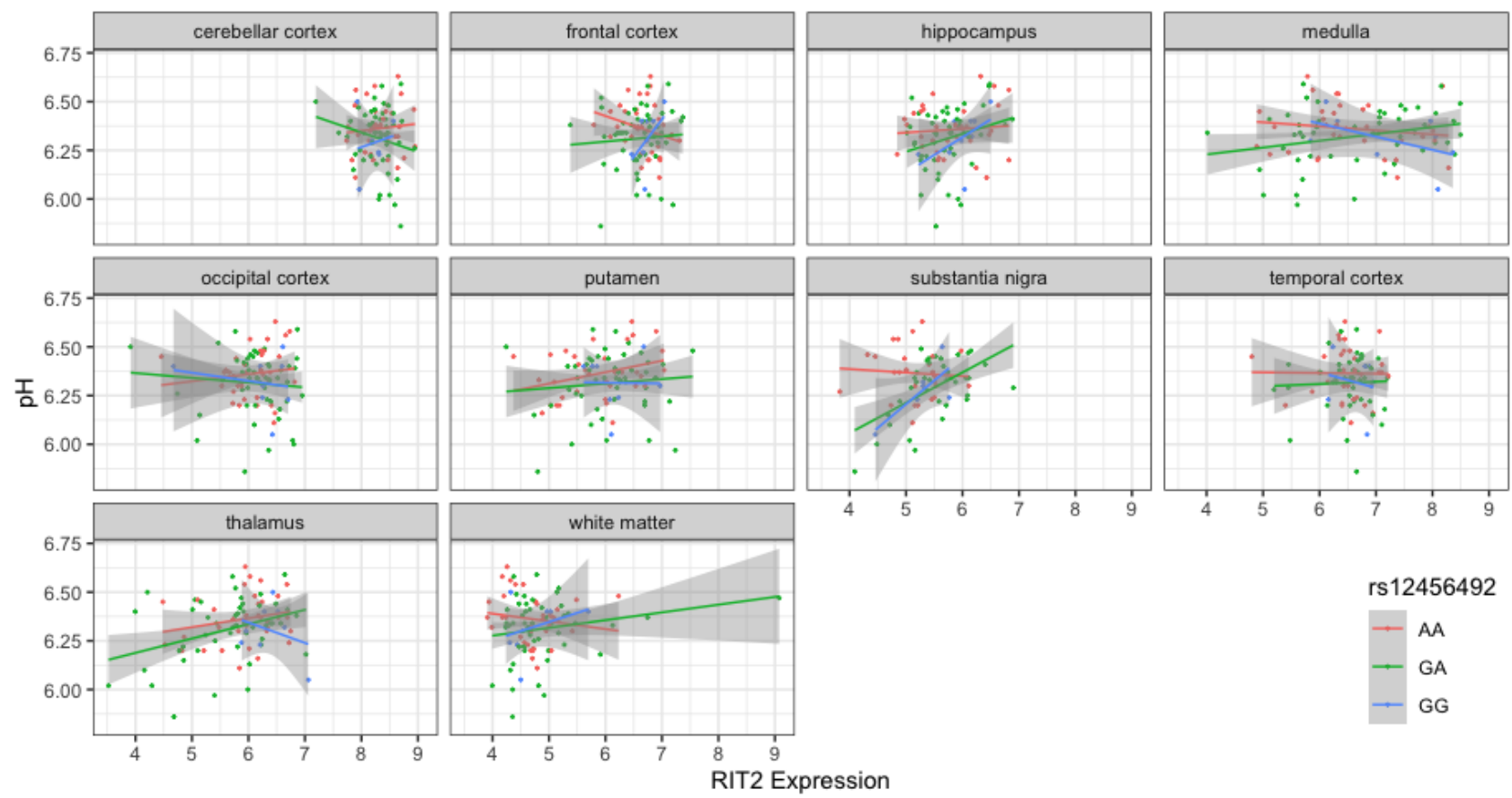

rs12456492

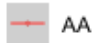

$\div$ GA

RIT2 Expression

Figure 2. Scatter plot of pH and RIT2 gene expression based on rs12456492 genotype for 10 brain regions. Genotype groups are colored with lines representing linear fits with shaded areas marking the 0.95 confidence interval. 

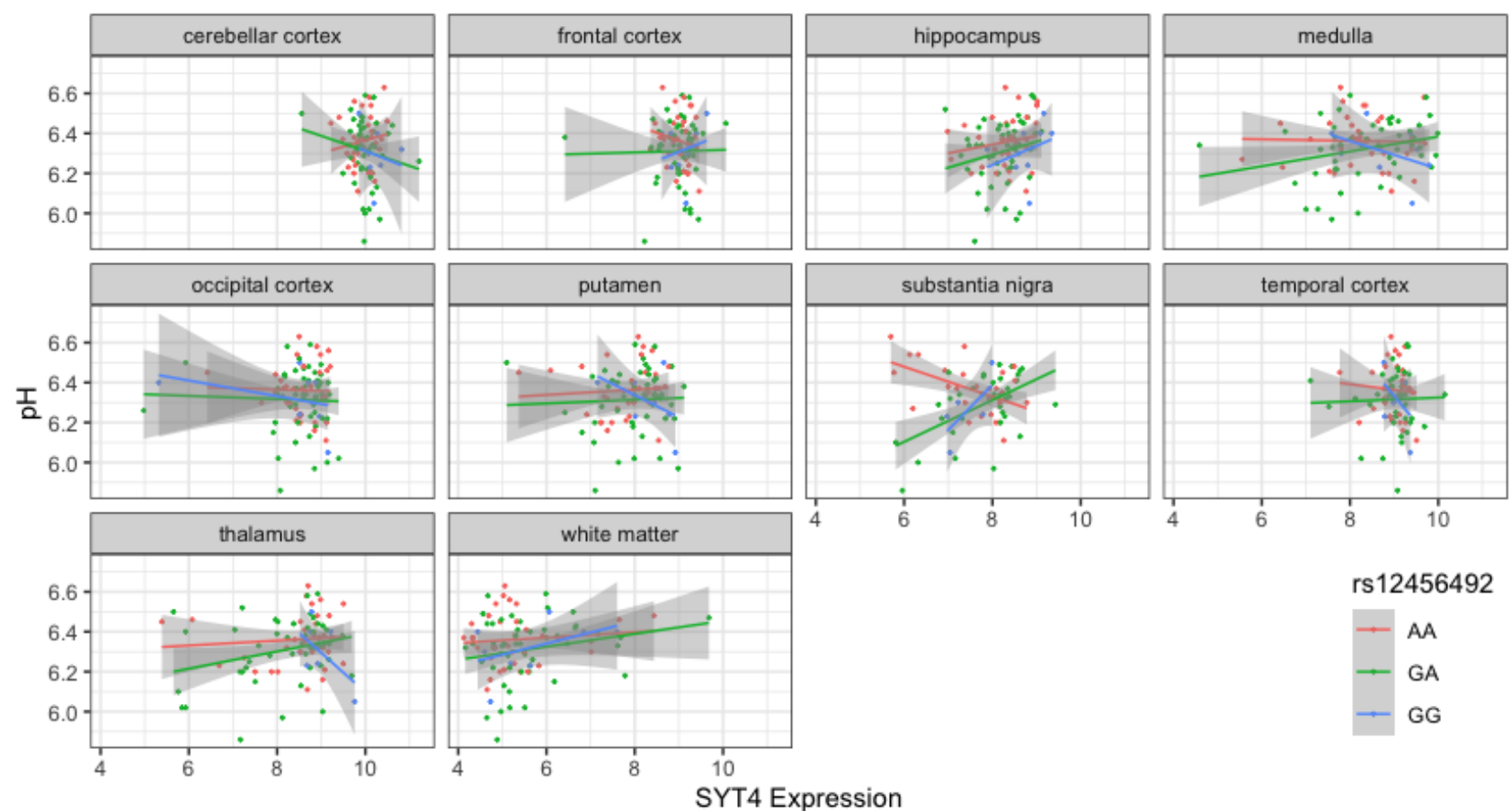

rs12456492

SYT4 Expression

Figure 3. Scatter plot of $\mathrm{pH}$ and SYT4 gene expression based on rs12456492 genotype for 10 brain regions. Genotype groups are colored with lines representing linear fits with shaded areas marking the 0.95 confidence interval.

To investigate the combined influences of RIT2 and SYT4 on pH level, we tested three additional models (Table 1, models 4-6). After including all covariates, the addition of SYT4 gene expression resulted in a slightly better fit in the RIT2 interaction model (Model 2 vs $4, R^{2}$ from 0.2 to 0.22 , $\mathrm{p}=0.11$ ). Similarly, the addition of RIT2 expression explained slightly more variance in the SYT4 interaction model (Model 3 vs $5, R^{2}$ from 0.28 to $0.33, p=0.02$ ). Models that have the SYT4 genetic interaction explain more variance than those that include the RIT2 interaction. Furthermore, in a model with all tested therms, the RIT2 genetic interaction term is no longer significant (Model 6).

Table 1: Effects of Rs12456492, RIT2, and SYT4 gene expression on brain pH across six models.

\begin{tabular}{|c|c|c|c|c|c|c|}
\hline & Model 1 & Model 2 & Model 3 & Model 4 & Model 5 & Model 6 \\
\hline $\begin{array}{l}\text { Rs12456492 } \\
\text { (SNP) }\end{array}$ & $\begin{array}{l}0.06 \\
(0.006-0.12) \\
p<0.04\end{array}$ & $\begin{array}{l}0.94 \\
(0.35-1.53), \\
p<0.003)\end{array}$ & $\begin{array}{l}1.38 \\
(0.84-1.92) \\
p<0.00001\end{array}$ & $\begin{array}{l}0.90 \\
(0.31-1.49) \\
p<0.004\end{array}$ & $\begin{array}{l}1.23 \\
(0.71-1.77) \\
p<0.00002\end{array}$ & $\begin{array}{l}1.23 \\
(0.64-1.81) \\
p<0.0001\end{array}$ \\
\hline RIT2 & & $\begin{array}{l}0.30 \\
(0.14-0.45), \\
p<0.0004\end{array}$ & & $\begin{array}{l}0.34 \\
(0.18-0.51) \\
p<0.0001\end{array}$ & $\begin{array}{l}0.11 \\
(0.02-0.20) \\
p<0.03\end{array}$ & $\begin{array}{l}0.10 \\
(-0.12-0.32) \\
p<0.37\end{array}$ \\
\hline SYT4 & & & $\begin{array}{l}0.26 \\
(0.16-0.37) \\
p<0.00001\end{array}$ & $\begin{array}{l}-0.05 \\
(-0.12-0.01) \\
p=0.11\end{array}$ & $\begin{array}{l}0.17 \\
(0.05-0.30) \\
p<0.006\end{array}$ & $\begin{array}{l}0.18 \\
(0.02-0.34) \\
p<0.03\end{array}$ \\
\hline RIT2×SNP & & $\begin{array}{l}-0.17 \\
(-0.23- \\
-0.05)\end{array}$ & & $\begin{array}{l}-0.16 \\
(-0.27--0.04) \\
p<0.007\end{array}$ & & $\begin{array}{l}0.01 \\
(-0.14-0.15) \\
p=0.93\end{array}$ \\
\hline
\end{tabular}




\begin{tabular}{|l|l|l|l|l|l|l|}
\hline & $p<0.005$ & & & & \\
\hline & & & $\begin{array}{l}-0.17 \\
(-0.24--0.10),\end{array}$ & & -0.15 & -0.16 \\
SYT4×SNP & & & $\begin{array}{l}(-0.22-0.08), \\
(-0.26-0.0001\end{array}$ & & $p<0.00006$ & $p<0.003$ \\
\hline Adjusted R2 & 0.013 & 0.20 & 0.28 & 0.22 & 0.33 & 0.32 \\
\hline
\end{tabular}

${ }^{*}$ After adjusting for sex, age, PMI and RIN

\section{Sex-specific signals}

To investigate if sex influenced the $\mathrm{pH}-\mathrm{eQTL}$ interaction we tested a three-way interaction between gene expression, genotype, and gender. This added interaction term was not significant for RIT2 $(B=0.21[-0.05-0.48], p=0.11)$, but was for SYT4 $(B=0.18$ [0.006 - 0.35], $p<0.042)$ when added to Models 2 and 3 . We additionally stratified our analyses to test for sex-specific effects on Model 2 and Model 3 (Figure 4). This yielded 54 male and a small group of 16 female samples that lack individuals carrying the GG genotype. In both models, $\mathrm{pH}$-eQTL interactions were significant for males (RIT2: $\mathrm{B}=-0.21, \mathrm{p}<0.0001$; SYT4: $\mathrm{B}=-0.21, \mathrm{p}<0.003)$ but not females $(R I T 2: \mathrm{B}=-0.03$, $\mathrm{p}=0.88$; SYT4: $\mathrm{B}=-0.09, \mathrm{p}=0.47)$. Overall, the SYT4 $\mathrm{pH}-\mathrm{eQTL}$ appears to be sex-specific, but this analysis is limited by sample size.
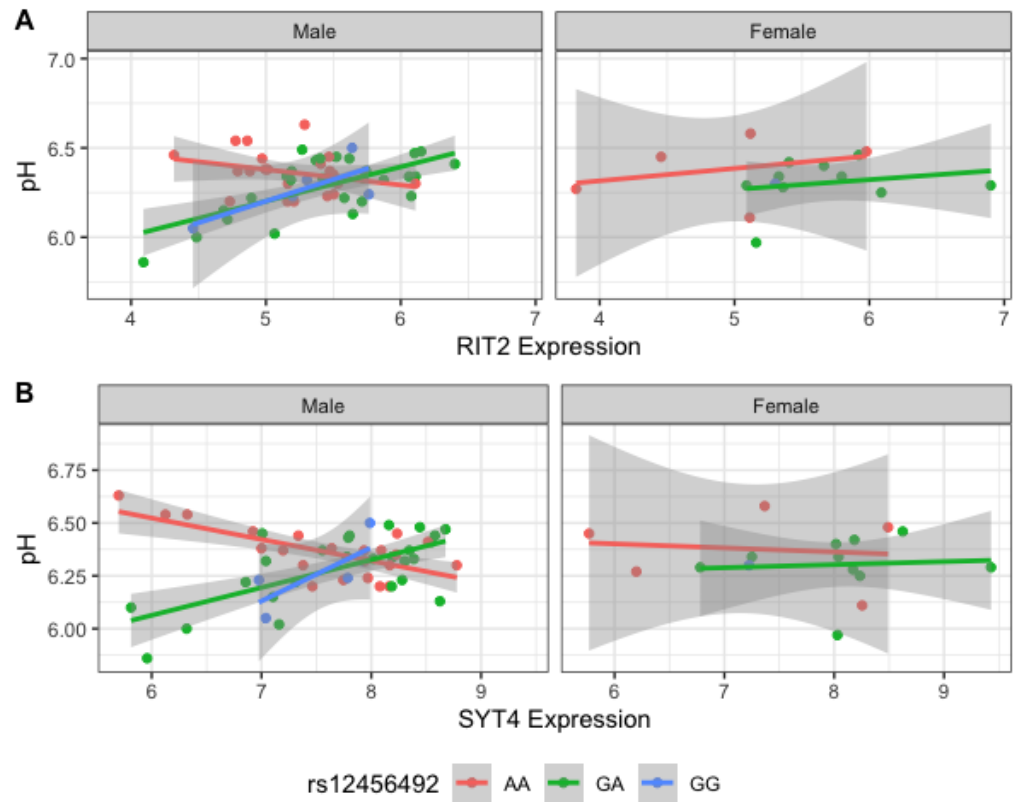

Figure 4: Scatter plot of $\mathrm{pH}$ and gene expression grouped by risk SNP genotype and stratified by sex within BrainEac sample for RIT2 (A) and SYT4 (B). Genotype groups are colored with lines representing linear fits with shaded areas marking the 0.95 confidence interval. 


\section{Rs12456492 is not associated with RIT2 and SYT4 expression and $\mathrm{pH}$ within the substantia nigra in the GTEx dataset}

Next, we used the GTEx dataset to test for replication of our findings from the BrainEac sample. We obtained gene expression data for RIT2 and SYT4 in the substantia nigra region, along with genotype data for the PD risk SNP rs12456492 yielding a sample size of 113 . The $\mathrm{pH}$ ranged from 5.58 to 6.79 , with a mean of 6.20 . Similarly, we removed $\mathrm{pH}$ outliers from the samples using the same criteria as done with the BrainEAC dataset, resulting in 104 samples.

Correlation between gene expression of RIT2 or SYT4 with $\mathrm{pH}$ was significant (RIT2: $\mathrm{r}=0.27, \mathrm{p}$ <0.006; SYT4: $r=0.31, p<0.002$ ). Next, we applied linear models on the GTEx data to first examine the influence of the risk SNP on $\mathrm{pH}$ level. The risk SNP by itself was not a significant predictor of $\mathrm{pH}$ $(p=0.14)$. Furthermore interaction between $\mathrm{pH}$, rs12456492 and either RIT2 or STY4 was not observed (RIT2: t-stat $=-1.10, p=0.28$, SYT4: $t$-stat $=-0.84, p=0.40)$. After accounting for the covariates (sex, age, RIN and PMI), the gene expression and SNP interactions were still not statistically significant terms in the models. Also, sex-specific signals were not observed in the GTEx substantia nigra samples.

\section{Shorter PMI values in GTEx}

To explain the failed replication in the GTEx dataset, we examined the differences between the two cohorts. We noticed that PMI was longer in BrainEAC when compared to the GTEx samples. For the BrainEac, PMI ranged from 31 to 99 hours, whereas GTEx ranged from 4.78 to 23.13 hours. Hence there was no overlap between the two datasets as seen in Figure 5.

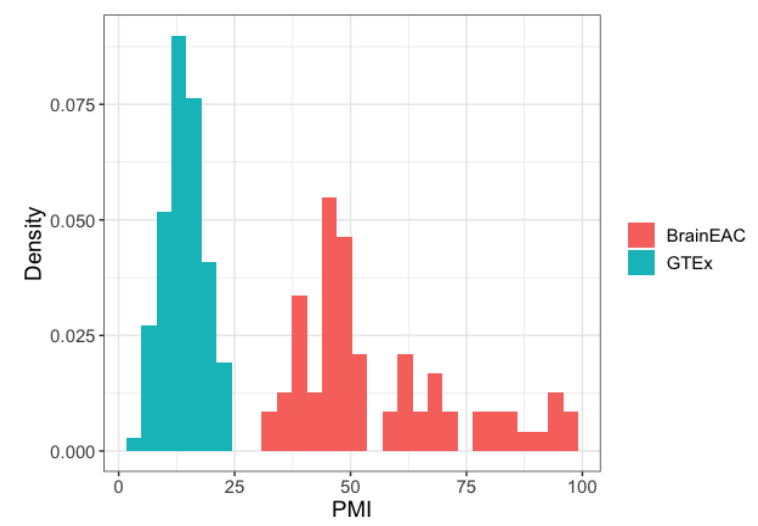

Figure 5. Density plot of PMI values in hours. GTEx and BrainEac samples are colored in blue and red, respectively.

\section{PMI influences $\mathrm{pH}$-eQTL strength}

To further investigate this difference, we stratified BrainEAC based on PMI. We split the sample based on the median PMI (49 hours). Using Model 2 and 3, interactions between gene expression and genotype were not statistically significant in short PMI (RIT2: $p=0.15$; SYT4:p=0.24) but were in 
long PMI ( $p<0.01$ for both genes). The interaction between SNP, gene expression and PMI was not statistically significant for RIT2 $(B=-0.005$ [-0.012 - 0.003], $p=0.20)$, but was for SYT4 $(B=-0.004$ [-0.008 - -0.0007], p<0.02). In Figure 6, G allele carriers in the stratified short and long PMI group show a positive correlation between gene expression and $\mathrm{pH}$ level. Although the long PMI group lacks GG carriers, a switch from positive to negative correlation is observed when comparing the GA and AA groups. This suggests the PMI difference between the datasets may explain why the $\mathrm{pH}-\mathrm{eQTL}$ interaction was not observed in the GTEx sample.
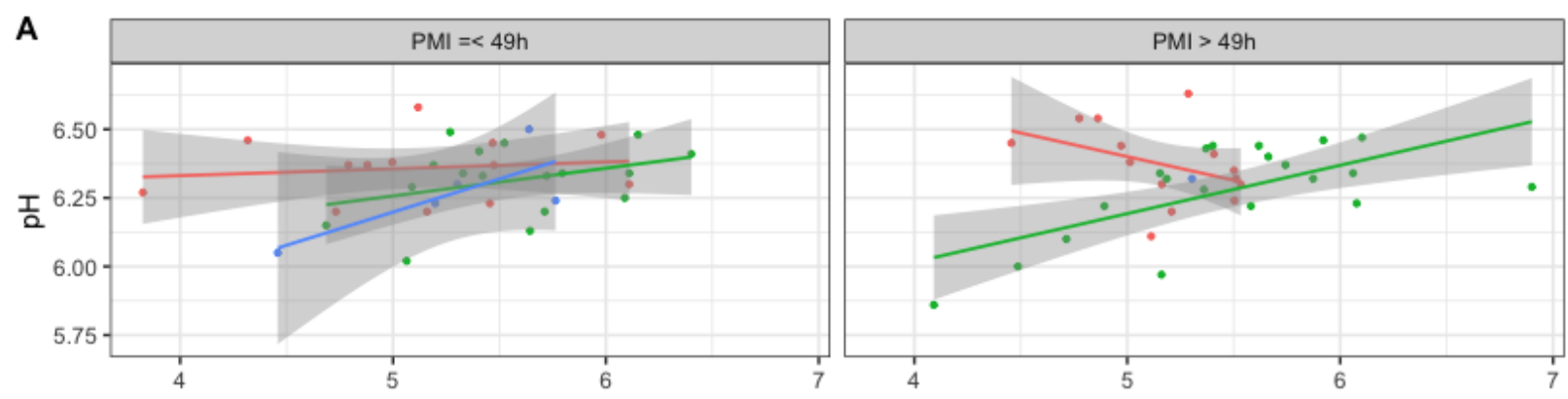

RIT2 Expression
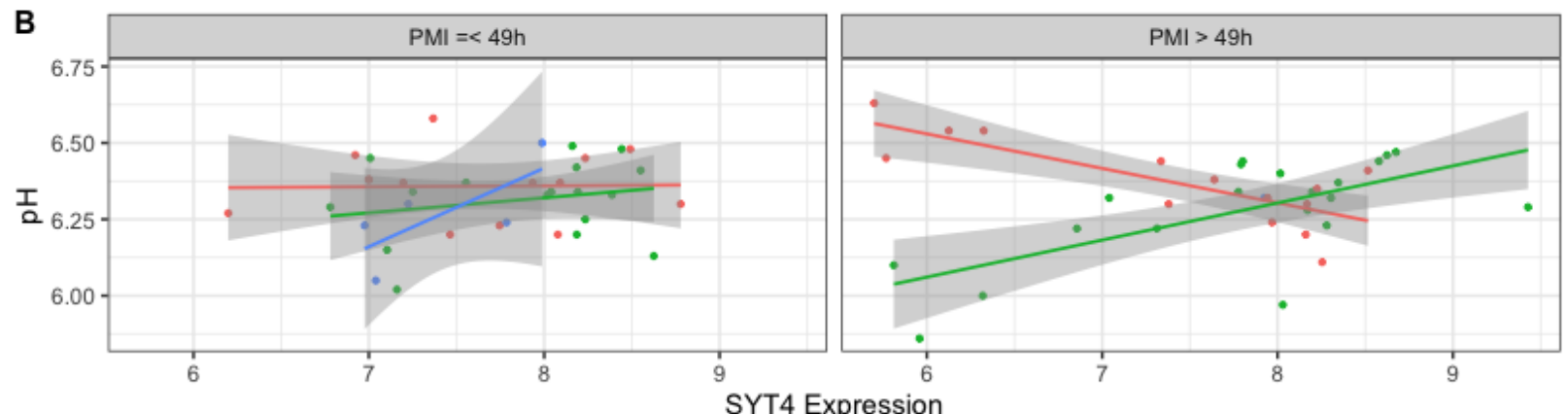

rs12456492

AA GA GG

Figure 6. Scatter plot of $\mathrm{pH}$ and gene expression grouped by risk SNP genotype and stratified PMI within BrainEac sample for RIT2 (A) and SYT4 (B). Genotype groups are colored with lines representing linear fits with shaded areas marking the 0.95 confidence interval.

\section{CA10 is co-expressed with SYT4 and RIT2}

Using a co-expression search tool, the two genes most co-expressed with RIT2 were carbonic anhydrase 10 (CA10) and SYT4. Of the 97 brain datasets that excluded cancer studies, based on co-expression, the most relevant dataset was a study of Parkinson's disease [GSE20146, (Zheng et al. 2010)]. In this top dataset that assayed expression in the globus pallidus interna ( $\mathrm{n}=19)$, RIT2 expression was correlated with CA10 ( $r=0.52, p<0.03)$ and SYT4 $(r=0.75, p<0.0003)$, but SYT4 was not co-expressed with $C A 10(r=0.43, p=0.065)$. We didn't observe differential co-expression when comparing cases and controls. We note that CA10 gene expression is not a significant predictor for $\mathrm{pH}$ when accounting for the other covariates (sex, age, PMI, and RIN) in either BrainEAC or GTEx substantia nigra samples. Therefore the addition of CA10 does not improve the models in predicting $\mathrm{pH}$. 


\section{Discussion}

This study replicates the positive correlation between $\mathrm{RIT2}$ gene expression and $\mathrm{pH}$ in the human brain. Furthermore, we found that a co-localized PD associated genetic variant altered this relationship between expression and $\mathrm{pH}$. When tested across ten brain regions, this interaction is specifically found in the substantia nigra, the primary location of Parkinson's disease pathology. A similar association was found for the neighbouring SYT4 gene. For carriers of the protective allele, the brain-wide positive correlation between gene expression and $\mathrm{pH}$ is inverted in the substantia nigra. In a combined model with both genes, the SYT4 relationship is stronger. We attempted to replicate these findings using the GTEx dataset. However, the association between SNP interaction and gene expression of either SYT4 and RIT2 with pH was not seen. We observed that the PMI values were longer in the BrainEac cohort compared to the GTEx cohort. After stratifying the BrainEac data into short and long PMI, we only observed the relationship in the longer PMI group. These associations implicate interactions between rs12456492, RIT2, and SYT4 in regulating $\mathrm{pH}$ in the brain.

When stratified by sex, the $\mathrm{pH}-\mathrm{eQTL}$ relationship was not observed in females. This was more evident for SYT4 than RIT2. The prevalence of PD is higher in males than females (Marras et al. 2018). Although our analysis was limited by sample size, we postulate that this sex-specific effect may help understand differences in PD incidence.

We suspect that we did not validate our findings in the GTEx dataset because of short PMI values in comparison to the BrainEAC samples. The differences between the datasets are clear, as there is no overlap between the PMI values. When the BrainEac cohort is split into short and long PMI groups, the results replicate the cohort differences. Specifically, the $\mathrm{pH}-\mathrm{eQTL}$ relationship is observed in the samples with long but not short PMI. In agreement, studies of postmortem gene expression have found gene, and genotype-dependent associations with PMI (Zhu et al. 2017; Scott et al. 2020). Although we performed our analyses on neuropathologically normal brains, we speculate that a longer PMI value represents a neurodegenerative state that is closer to a Parkinsonian brain. Follow-up studies of postmortem samples or cell cultures derived from PD patients are warranted to test our findings in an experimental setting.

Several studies have examined pH-dependent interactions in the context of Parkinson's disease. For example, $\alpha$-synuclein aggregation and stability are increased at acidic $\mathrm{pH}$ (Lv et al. 2016; Pham et al. 2009). In addition, $\beta$-synuclein, an inhibitor of $\alpha$-synuclein aggregation, is sensitive to $\mathrm{pH}$ and forms fibrils in mildly acidic pH (Moriarty et al. 2017; Williams et al. 2018). Using quantum chemical methods, Umek and colleagues determined that an acidic environment is required to prevent dopamine autoxidation (Umek et al. 2018). Kinetic modelling has also revealed that $\mathrm{pH}$ interactions with iron and dopamine could lead to oxidative stress (Sun et al. 2018). Caffeine consumption is associated with mild alkalosis (Tajima 2010) and a decreased risk of PD (Costa et al. 2010). We also note that RIT2 is differentially co-expressed with interferon-gamma signalling genes in substantia nigra samples from PD cases (Liscovitch and French 2014). While indirect, interferon-gamma is acid-labile, and it's overexpression in mice causes nigrostriatal neurodegeneration (Piasecki 1999; Chakrabarty et al. 2011). Mitochondria, which internally maintain an alkaline $\mathrm{pH}$, are thought to be dysfunctional in PD (Chen, Turnbull, and Reeve 2019). 
Mitochondrial dysfunction can lead to oxidative stress, resulting in lactic and intraneuronal acidosis (Arias et al. 2008; Koga et al. 2006; Balut et al. 2008). Recently, Rango and colleagues found that carriers of PINK1 mutations, which are associated with early-onset PD, have abnormal pH levels in the visual cortex. Specifically, carriers of homozygous PINK1 mutations had a higher $\mathrm{pH}$ at rest when compared to healthy controls and patients without PINK1 mutations. Unlike healthy controls, $\mathrm{pH}$ did not increase upon activation in homozygous PINK1 mutation carriers (Rango et al. 2020). Taken together, future studies of RIT2 and SYT4 that examine $\mathrm{pH}$ in the context of interferon-gamma, dopamine autoxidation, caffeine consumption, and mitochondrial function are warranted.

Both SYT4 and Carbonic Anhydrase 10 (CA10) are co-expressed with RIT2. Unlike RIT2 and SYT4, carbonic anhydrases are known to regulate intracellular and extracellular pH (Wingo et al. 2001). However, CA10 has been found to be catalytically inactive and forms complexes with synaptic proteins (Sterky et al. 2017; Sjöblom et al. 1996; Nishimori et al. 2013). In agreement, CA10 gene expression was not a predictor for brain $\mathrm{pH}$ when added to either the RIT2 or SYT4 models. Recently, Payan-Gomez and colleagues performed a co-expression network analysis of the human prefrontal cortex. In this analysis that used brain samples from old and young individuals, CA10 was identified as a central gene in the network (Payán-Gómez et al. 2019). A related gene, CA2, was found to be elevated in mitochondria from middle-aged mouse brain samples (Pollard et al. 2016). Furthermore, carbonic anhydrase inhibitors have been found to prevent amyloid beta-induced mitochondrial toxicity (Solesio et al. 2018; Fossati et al. 2016). Further study of interactions between RIT2 and CA10 may reveal possible $\mathrm{pH}$ regulation mechanisms that are relevant to $P D$.

\section{Conclusion}

This study described a relationship between gene expression and $\mathrm{pH}$ that interacted with genetic variation. Our analysis of this $\mathrm{pH}-\mathrm{eQTL}$ relationship is constrained to the RIT2 locus that is strongly associated with PD risk and is only observed in substantia nigra. Additional interactions with sex and PMI were observed for SYT4 and, to a lesser degree, RIT2. These previously unknown associations suggest new mechanistic roles for rs12456492, RIT2, and SYT4 in the Parkinsonian brain.

\section{Acknowledgements}

We thank the GTEx team for adding brain $\mathrm{pH}$ to the database. We thank Drs. Suneil Kalia and Lorraine Kalia for their insightful comments and suggestions.

This study was supported by the CAMH Foundation, CAMH Discovery Fund, and a National Science and Engineering Research Council of Canada (NSERC) Discovery Grant to LF. 


\section{Competing Interests}

LF owns shares in Cortexyme Inc., a company that is developing treatments for neurodegenerative disease. The other authors declare no conflict of interest.

\section{References}

Arias, Robert L., Mei-Li A. Sung, Dmytro Vasylyev, Mei-Yi Zhang, Kristin Albinson, Katie Kubek, Natasha Kagan, et al. 2008. "Amiloride Is Neuroprotective in an MPTP Model of Parkinson's Disease." Neurobiology of Disease 31 (3): 334-41.

Balut, C., M. vandeVen, S. Despa, I. Lambrichts, M. Ameloot, P. Steels, and I. Smets. 2008. "Measurement of Cytosolic and Mitochondrial pH in Living Cells during Reversible Metabolic Inhibition." Kidney International 73 (2): 226-32.

Bellou, Vanesa, Lazaros Belbasis, loanna Tzoulaki, Evangelos Evangelou, and John P. A. Ioannidis. 2016. "Environmental Risk Factors and Parkinson's Disease: An Umbrella Review of Meta-Analyses." Parkinsonism \& Related Disorders 23 (February): 1-9.

Chakrabarty, Paramita, Carolina Ceballos-Diaz, Wen-Lang Lin, Amanda Beccard, Karen Jansen-West, Nikolaus R. McFarland, Christopher Janus, Dennis Dickson, Pritam Das, and Todd E. Golde. 2011. "Interferon-y Induces Progressive Nigrostriatal Degeneration and Basal Ganglia Calcification." Nature Neuroscience 14 (6): 694-96.

Chang, Diana, Mike A. Nalls, Ingileif B. Hallgrímsdóttir, Julie Hunkapiller, Marcel van der Brug, Fang Cai, International Parkinson's Disease Genomics Consortium, et al. 2017. "A Meta-Analysis of Genome-Wide Association Studies Identifies 17 New Parkinson's Disease Risk Loci." Nature Genetics 49 (10): 1511-16.

Chen, Chun, Doug M. Turnbull, and Amy K. Reeve. 2019. "Mitochondrial Dysfunction in Parkinson's Disease-Cause or Consequence?" Biology 8 (2). https://doi.org/10.3390/biology8020038.

Core, R. 2013. "Team RA Language and Environment for Statistical Computing: Vienna, Austria. 2013."

Costa, João, Nuno Lunet, Catarina Santos, João Santos, and António Vaz-Carneiro. 2010. "Caffeine Exposure and the Risk of Parkinson's Disease: A Systematic Review and Meta-Analysis of Observational Studies." Journal of Alzheimer's Disease: JAD 20 Suppl 1: S221-38.

Dean, Camin, Huisheng Liu, F. Mark Dunning, Payne Y. Chang, Meyer B. Jackson, and Edwin R. Chapman. 2009. "Synaptotagmin-IV Modulates Synaptic Function and Long-Term Potentiation by Regulating BDNF Release." Nature Neuroscience 12 (6): 767-76.

Do, Chuong B., Joyce Y. Tung, Elizabeth Dorfman, Amy K. Kiefer, Emily M. Drabant, Uta Francke, Joanna L. Mountain, et al. 2011. "Web-Based Genome-Wide Association Study Identifies Two Novel Loci and a Substantial Genetic Component for Parkinson's Disease." PLoS Genetics 7 (6): e1002141.

Edgar, Ron, Michael Domrachev, and Alex E. Lash. 2002. "Gene Expression Omnibus: NCBI Gene Expression and Hybridization Array Data Repository." Nucleic Acids Research 30 (1): 207-10.

Fossati, Silvia, Patrizia Giannoni, Maria E. Solesio, Sarah L. Cocklin, Erwin Cabrera, Jorge Ghiso, and Agueda Rostagno. 2016. "The Carbonic Anhydrase Inhibitor Methazolamide Prevents Amyloid Beta-Induced Mitochondrial Dysfunction and Caspase Activation Protecting Neuronal and Glial Cells in Vitro and in the Mouse Brain." Neurobiology of Disease 86 (February): 29-40.

Hwang, Onyou. 2013. "Role of Oxidative Stress in Parkinson's Disease." Experimental Neurobiology 22 (1): 11-17.

Koga, Keiko, Atsushi Mori, Satoshi Ohashi, Naoki Kurihara, Hisashi Kitagawa, Makoto Ishikawa, 
Yasuhide Mitsumoto, and Masami Nakai. 2006. "1H MRS Identifies Lactate Rise in the Striatum of MPTP-Treated C57BL/6 Mice.” The European Journal of Neuroscience 23 (4): 1077-81.

Lim, Nathaniel, Stepan Tesar, Manuel Belmadani, Guillaume Poirier-Morency, Burak Ogan Mancarci, Jordan Sicherman, Matthew Jacobson, Justin Leong, Patrick Tan, and Paul Pavlidis. 2021. "Curation of over 10000 Transcriptomic Studies to Enable Data Reuse." Database: The Journal of Biological Databases and Curation 2021 (February).

https://doi.org/10.1093/database/baab006.

Liscovitch, Noa, and Leon French. 2014. "Differential Co-Expression between a-Synuclein and IFN-y Signaling Genes across Development and in Parkinson's Disease." PloS One 9 (12): e115029.

Lonsdale, John, Jeffrey Thomas, Mike Salvatore, Rebecca Phillips, Edmund Lo, Saboor Shad, Richard Hasz, et al. 2013. "The Genotype-Tissue Expression (GTEx) Project." Nature Genetics 45 (6): 580-85.

Lv, Zhengjian, Alexey V. Krasnoslobodtsev, Yuliang Zhang, Daniel Ysselstein, Jean Christophe Rochet, Scott C. Blanchard, and Yuri L. Lyubchenko. 2016. "Effect of Acidic pH on the Stability of a-Synuclein Dimers." Biopolymers 105 (10): 715-24.

Marras, C., J. C. Beck, J. H. Bower, E. Roberts, B. Ritz, G. W. Ross, R. D. Abbott, et al. 2018. "Prevalence of Parkinson's Disease across North America." NPJ Parkinson's Disease 4 (July): 21.

Mendez, Jose Alfredo, Marie-Josée Bourque, Caroline Fasano, Christian Kortleven, and Louis-Eric Trudeau. 2011. "Somatodendritic Dopamine Release Requires Synaptotagmin 4 and 7 and the Participation of Voltage-Gated Calcium Channels." The Journal of Biological Chemistry 286 (27): 23928-37.

Millar, T., R. Walker, J-C Arango, J. W. Ironside, D. J. Harrison, D. J. Maclntyre, D. Blackwood, C. Smith, and J. E. Bell. 2007. "Tissue and Organ Donation for Research in Forensic Pathology: The MRC Sudden Death Brain and Tissue Bank." The Journal of Pathology 213 (4): 369-75.

Mistry, M., and P. Pavlidis. 2010. "A Cross-Laboratory Comparison of Expression Profiling Data from Normal Human Postmortem Brain." Neuroscience 167 (2): 384-95.

Moriarty, Gina M., Michael P. Olson, Tamr B. Atieh, Maria K. Janowska, Sagar D. Khare, and Jean Baum. 2017. "A pH-Dependent Switch Promotes $\beta$-Synuclein Fibril Formation via Glutamate Residues." The Journal of Biological Chemistry 292 (39): 16368-79.

Nalls, Mike A., Cornelis Blauwendraat, Costanza L. Vallerga, Karl Heilbron, Sara Bandres-Ciga, Diana Chang, Manuela Tan, et al. 2019. "Identification of Novel Risk Loci, Causal Insights, and Heritable Risk for Parkinson's Disease: A Meta-Analysis of Genome-Wide Association Studies." Lancet Neurology 18 (12): 1091-1102.

Nishimori, Isao, Daniela Vullo, Tomoko Minakuchi, Andrea Scozzafava, Clemente Capasso, and Claudiu T. Supuran. 2013. "Restoring Catalytic Activity to the Human Carbonic Anhydrase (CA) Related Proteins VIII, X and XI Affords Isoforms with High Catalytic Efficiency and Susceptibility to Anion Inhibition." Bioorganic \& Medicinal Chemistry Letters 23 (1): 256-60.

Pankratz, Nathan, Gary W. Beecham, Anita L. DeStefano, Ted M. Dawson, Kimberly F. Doheny, Stewart A. Factor, Taye H. Hamza, et al. 2012. "Meta-Analysis of Parkinson's Disease: Identification of a Novel Locus, RIT2." Annals of Neurology 71 (3): 370-84.

Payán-Gómez, César, Julián Riaño-Moreno, Diana Amador-Muñoz, and Sandra Ramírez-Clavijo. 2019. "Co-Expression Network Analysis Identifies Possible Hub Genes in Aging of the Human Prefrontal Cortex." Revista Ciencias de La Salud 17 (2): 201-22.

Pham, Chi L. L., Su Ling Leong, Feda E. Ali, Vijaya B. Kenche, Andrew F. Hill, Sally L. Gras, Kevin J. Barnham, and Roberto Cappai. 2009. "Dopamine and the Dopamine Oxidation Product 5,6-Dihydroxylindole Promote Distinct On-Pathway and Off-Pathway Aggregation of a-Synuclein in a pH-Dependent Manner." Journal of Molecular Biology. https://doi.org/10.1016/j.jmb.2009.02.007.

Piasecki, E. 1999. "Human Acid-Labile Interferon Alpha." Archivum Immunologiae et Therapiae Experimentalis 47 (2): 89-98. 
Pollard, Amelia, Freya Shephard, James Freed, Susan Liddell, and Lisa Chakrabarti. 2016. "Mitochondrial Proteomic Profiling Reveals Increased Carbonic Anhydrase II in Aging and Neurodegeneration." Aging 8 (10): 2425-36.

Ramasamy, Adaikalavan, Daniah Trabzuni, Sebastian Guelfi, Vibin Varghese, Colin Smith, Robert Walker, Tisham De, et al. 2014. "Genetic Variability in the Regulation of Gene Expression in Ten Regions of the Human Brain." Nature Neuroscience 17 (10): 1418-28.

Rango, Mario, Gabriele Dossi, Letizia Squarcina, and Cristiana Bonifati. 2020. "Brain Mitochondrial Impairment in Early-Onset Parkinson's Disease with or without PINK1 Mutation." Movement Disorders: Official Journal of the Movement Disorder Society 35 (3): 504-7.

Scott, Latia, Sheree J. Finley, Clytrice Watson, and Gulnaz T. Javan. 2020. "Life and Death: A Systematic Comparison of Antemortem and Postmortem Gene Expression." Gene 731 (March): 144349.

Sinning, Anne, and Christian A. Hübner. 2013. "Minireview: pH and Synaptic Transmission." FEBS Letters 587 (13): 1923-28.

Sjöblom, B., B. Elleby, K. Wallgren, B. H. Jonsson, and S. Lindskog. 1996. "Two Point Mutations Convert a Catalytically Inactive Carbonic Anhydrase-Related Protein (CARP) to an Active Enzyme." FEBS Letters 398 (2-3): 322-25.

Solesio, María E., Pablo M. Peixoto, Ludovic Debure, Stephen M. Madamba, Mony J. de Leon, Thomas Wisniewski, Evgeny V. Pavlov, and Silvia Fossati. 2018. "Carbonic Anhydrase Inhibition Selectively Prevents Amyloid $\beta$ Neurovascular Mitochondrial Toxicity." Aging Cell 17 (4): e12787.

Sterky, Fredrik H., Justin H. Trotter, Sung-Jin Lee, Christian V. Recktenwald, Xiao Du, Bo Zhou, Peng Zhou, Jochen Schwenk, Bernd Fakler, and Thomas C. Südhof. 2017. "Carbonic Anhydrase-Related Protein CA10 Is an Evolutionarily Conserved Pan-Neurexin Ligand." Proceedings of the National Academy of Sciences of the United States of America 114 (7): E1253-62.

Sun, Yingying, A. Ninh Pham, Dominic J. Hare, and T. David Waite. 2018. "Kinetic Modeling of $\mathrm{pH}$-Dependent Oxidation of Dopamine by Iron and Its Relevance to Parkinson's Disease." Frontiers in Neuroscience 12 (November): 859.

Tajima, Yutaka. 2010. "Coffee-Induced Hypokalaemia." Clinical Medicine Insights. Case Reports 3 (April): 9-13.

Trabzuni, Daniah, Mina Ryten, Robert Walker, Colin Smith, Sabaena Imran, Adaikalavan Ramasamy, Michael Weale, and John Hardy. 2012. "Quality Control Parameters on a Large Dataset of Regionally Dissected Human Control Brains for Whole Genome Expression Studies." Journal of Neurochemistry. https://doi.org/10.1111/j.1471-4159.2011.07602.x.

Umek, Nejc, Blaž Geršak, Neli Vintar, Maja Šoštarič, and Janez Mavri. 2018. "Dopamine Autoxidation Is Controlled by Acidic pH." Frontiers in Molecular Neuroscience 11 (December): 467.

Williams, Jonathan K., Xue Yang, Tamr B. Atieh, Michael P. Olson, Sagar D. Khare, and Jean Baum. 2018. "Multi-Pronged Interactions Underlie Inhibition of $\alpha$-Synuclein Aggregation by ß-Synuclein." Journal of Molecular Biology 430 (16): 2360-71.

Wingo, T., C. Tu, P. J. Laipis, and D. N. Silverman. 2001. "The Catalytic Properties of Human Carbonic Anhydrase IX." Biochemical and Biophysical Research Communications 288 (3): 666-69.

Zheng, Bin, Zhixiang Liao, Joseph J. Locascio, Kristen A. Lesniak, Sarah S. Roderick, Marla L. Watt, Aron C. Eklund, et al. 2010. "PGC-1a, a Potential Therapeutic Target for Early Intervention in Parkinson's Disease." Science Translational Medicine 2 (52): 52 ra73.

Zhu, Yizhang, Likun Wang, Yuxin Yin, and Ence Yang. 2017. "Systematic Analysis of Gene Expression Patterns Associated with Postmortem Interval in Human Tissues." Scientific Reports 7 (1): 5435. 